\title{
Correlation between Antimicrobial Resistance and Nosocomial Infections, their Prevention and Control: Review
}

\author{
Sedhain Arpan, Roy Kalyan*, Chakraborty Mainak, Gauthaman Karunakaran \\ Department of Pharmacology, Himalayan Pharmacy Institute, Sikkim University, Sikkim. INDIA.
}

\begin{abstract}
Nosocomial infections (NI) are infections acquired by a patient during hospital stay. They cause a surge in morbidity, mortality, prolonged hospital stay and increased health care costs and also increase antibiotic use, leading to the development of antimicrobial resistance in the hospital. Nosocomial microorganisms are transmitted from person to person, environment and contaminated healthcare personnel. Nosocomial infections are the important preventable cause of morbidity and mortality. Approximately one-third of these infections can be prevented by sticking to established infection control guidelines. Nosocomial infections can be controlled by the coordination among healthcare professionals in formulating, implementing and with compliance towards policies related to the prevention and control of
\end{abstract}

infections and the use of antibiotics.

Key words: Nosocomial infections, Antimicrobial resistance, Infection prevention.

Correspondence

Dr. Kalyan Roy

Department of Pharmacology, Himalayan Pharmacy Institute, Sikkim University, Sikkim. INDIA

Phone: +919800083936

Email: roykalyan2005@gmail.com

DOI: $10.5530 /$ jyp.2020.12.6

\section{INTRODUCTION}

Nosocomial infections (NIs) are a great challenge for hospital personnel. Infection control is an acute issue in clinical setup for patient's health and safety. A nosocomial term is used for any disease acquired by a patient under medical care. It is an infection acquired by a patient during hospital stay. ${ }^{1}$ Recently, a new term, "healthcare-associated infections" (HCAI) is used for the infections caused by the prolonged hospital stay and is a significant risk factor for serious health issues leading to death. ${ }^{2}$ Nosocomial infections pose grave public health problem in developing countries, as well as in developed countries. On average, Nosocomial infections affects at least $7 \%$ of patients admitted to hospital in high-income countries and about $15 \%$ of those in low-income and middle-income countries. ${ }^{3}$ Health Care-Associated Infection, causes a surge in morbidity, mortality, prolonged hospital stay and increased health care costs and also increase antibiotic use, antibiotic pressure leading to the development of antimicrobial resistance in the hospital. ${ }^{4}$ Nosocomial infections are the important preventable cause of morbidity and mortality. Approximately one-third of these infections can be prevented by sticking to established infection control guidelines. ${ }^{5}$

Asymptomatic patients may be considered infected if these pathogens are found in the body fluids or at a sterile body site, such as blood or cerebrospinal fluid. ${ }^{6}$ Infections that are acquired by hospital staffs, visitors or other healthcare personnel may also be considered as nosocomial. The situations in which infections are not regarded as nosocomial are: The infections that were present at the time of admission and become complicated, though pathogens or symptoms change resulting in a new infection; The infections that are attained trans-placentally due to some diseases like toxoplasmosis, rubella, syphilis and show symptoms $48 \mathrm{hr}$ after birth. ${ }^{7}$

Healthcare-associated infection (HAIs) and increased resistance to antimicrobials have necessitated infection prevention and control (IPC) at the significant point to improve quality of care. Effective methods for changing the IPC conducts of healthcare-workers (HCWs) are needed to reduce HAIs and improve patients' safety, although a little is studied in this area. ${ }^{8}$ The incidence of emerging infectious diseases in humans has been increasing now. The factors that cause infection are complex and there are no simple solutions to prevention or cure. However, with certain precautions, nosocomial infections could be reduced.

In this study, all the data were collected from search engines as follows: PubMed, ScienceDirect and Google Scholar. We used these keywords "Nosocomial infection", "Healthcare-associated infections", "Infection prevention and control" and "Antimicrobial resistance" during searching in database.

All references mentioned in this review article were written in English and from the time interval of 1973 to the present.

1) Hand Hygiene: At any time 1.4 million people worldwide are suffering from healthcare-associated infections. Transmission occurs mostly via the hands of health-care workers. ${ }^{9}$ Organisms reside on the patient's skin; or are shed on inanimate objects in and around the patient. Organisms are then shifted to the hands of HCWs; organisms survive on hands and are transferred back to the environment or another patient; cross-transmission of organisms by contaminated or incompletely cleaned hands leading to infection and outbreak. Hand hygiene, though simple and inexpensive, has been recognized as one of the single most important interventions to prevent cross-contamination. ${ }^{4}$ Despite, many health-care facilities having well-established policies for hand hygiene, compliance remains difficult to achieve and sustain. ${ }^{10}$ Adherence to the practice remains at around $40 \%$. Reasons for non-adherence include work pressure and unavailability of materials. Easy access to hand-rub solutions, education, training, monitoring, feedback and institutional commitment might contribute to staff sensitivity to hand hygiene 
practices. ${ }^{911}$ Prompting and reminding HCWs by patients and visitors whether they have cleaned their hands are helpful. ${ }^{12}$

With the occurrence of antibiotic-resistant organisms, the significance of hand hygiene within hospitals has further increased. ${ }^{11}$ Based on the WHO's "My five moments for hand hygiene" framework, the observation of HCWs is done in the following five areas: before patient contact; before aseptic/clean task; after bodily fluid exposure; after patient contact; after contact with a patient's surroundings. ${ }^{13}$ In addition to the hand sanitization, false fingernails, nail varnish, stoned rings and wrist watches increases the chances of bacterial transfer, contribute to outbreaks of infection and interfere with proper hand hygiene practices, thus are not to be worn by clinical staff, a large section of them continue to do so. ${ }^{14}$ In 2009, WHO Patient Safety launched an extension of the programme; "SAVE LIVES: Clean Your Hands", a drive that aims to ensure an ongoing global, regional, national and local focus on hand hygiene in healthcare. WHO advocates the use of alcohol-based hand rubs when hands are not visibly contaminated. Alcohol-based hand rubs are the only known means for rapidly and effectively inactivating a wide range of potentially harmful micro-organisms on hands. ${ }^{15}$

2) Impact of Barrier Precautions in Reducing the Transmission of Serious Nosocomial Infections: Many nosocomial infections are easily transferable from patient-to-patient, either via the hands of healthcare workers or through the contamination of inanimate objects, including clothing and equipment. ${ }^{16}$ For some infections, the threat to other patients is regarded serious enough that many institutions use special precautions, such as the use of gloves, gowns and disposable equipment for all patient contact, especially in caring for patients colonized or infected with pathogens such as Vancomycin-resistant enterococci (VRE) ${ }^{17}$ and Clostridium difficile. ${ }^{18}$ Health care workers must use gloves and gowns when entering the room of an infected or colonized patient and remove them upon leaving (followed immediately by hand washing) to reduce the chances of clothing or equipment contamination that could transmit pathogens to other patients. ${ }^{17}$ For patients colonized with VRE and/or C. difficile, hospital equipment (i.e. blood pressure cuffs, thermometers) should remain in a patient's room and not carried across rooms. Patients colonized or infected with similar pathogens should be admitted to specific floors of the hospital where designated health care workers care only for patients colonized or infected with these pathogens. ${ }^{18}$

3) Environmental hygiene Spread of nosocomial infections from pools of pathogens in the inanimate hospital environment, particularly air, surfaces and fomites is unclear and the value of routine microbiologic surveillance has been unclear. ${ }^{19}$ Presence of pathogens in the hospital environment, although necessary, is not sufficient to prove a causal role in the pathogenesis of nosocomial infections. ${ }^{19}$ In a study when a hospital moved from its old building into a new building, the examination of the correlation between environmental contamination and endemic nosocomial infections was done. Despite major differences in environmental contamination between the old and new hospitals, the incidences of nosocomial infections in patients remain unchanged. It was concluded that organisms in the inanimate hospital environment such as air, water, nozzles, sink drains contributed negligibly to endemic nosocomial infections. ${ }^{19}$

5) Although much about the spread of nosocomial infections remains unknown, data from another study revealed that: (1) inanimate environmental surfaces could become contaminated for long after exposure to colonized patients. (2) Although an organism may be endemic within an institution, specific isolates may dominate in the inanimate environment (for C. difficile and VRE); and
(3) contaminated rooms may be a risk factor for the acquisition of nosocomial pathogens by unaffected patients. A research carried out to test the survival and disinfection of parainfluenza viruses on environmental surfaces suggested that parainfluenza viruses can survive on non-absorptive surfaces for as long as $10 \mathrm{hr}$ in the moist environment and even after the surface is dried virus was recoverable for up to $2 \mathrm{hr}$. When materials absorbed the virus-containing fluid (hospital gown, laboratory coat and facial tissue), viruses could be recovered for up to $4 \mathrm{hr}$. It was further seen that treating or laundering hospital gowns might reduce the viability of virus found in patients' secretions. ${ }^{20}$ Airborne transmission may well be important since projectile vomiting could give rise to infectious aerosols. Air current generated by open windows or air conditioning will scatter aerosols widely. Moreover, when aerosols are released during projectile vomiting in confined spaces, inhalation of an aerosolized virus is likely. Projectile vomitors present an important source of the infectious virus and this poses great problems for control of infection procedures within hospitals. ${ }^{21}$

4) Screening and cohorting patients: Antibiotic-resistant organisms are a common source of hospital-acquired infections. Infections due to methicillin-resistant Staphylococcus aureus (MRSA) and VRE cause morbidity and mortality, prolonged hospital stay and increased costs of care when matched to antibiotic-susceptible strains. ${ }^{22}$ Early detection of patients colonized with MRSA and subsequent prevention of patient-to-patient spread through infection control measures can act as potent interventions to control MRSA. ${ }^{23}$ Because of the increased cost of caring for an MRSA patient, interventions that decrease the spread of infections-like screening intensive care unit patients upon admission are more economical. ${ }^{24}$ Patients with positive surveillance culture results should be placed under isolation precautions to prevent transmission by way of respiratory droplets. Surveillance at hospital admission for multidrug-resistant (MDR) gram-negative bacteria (GNB) is not often done, potentially leaving carriers of these organisms unrecognized and not placed in transmission precautions until they develop an infection. ${ }^{25}$ Data from a large number of hospitals suggest that MRSA present in the anterior nares can serve as a marker to identify patients with a higher likelihood of harboring MDR GNB. Thus when patients are placed in contact precautions because of a positive MRSA screen, there may be an added benefit of isolating patients who are at increased risk for spreading MDR GNB to others inside the hospital. ${ }^{26}$ Programs using active screening of the anterior nares for MRSA colonization have been linked with reduced MRSA transmissions and other related infections such as VRE and Clostridium difficile. Available rapid molecular tests and noninvasive sampling methods make screening for MRSA colonization comparatively easy compared with screening for other MDROs. Even in the set of a community hospital with a fairly low prevalence of MRSA (34\%), practice of an active surveillance program for MRSA was shown to be a cost-effective strategy, decreasing the risk of acquiring MRSA infection and preventing the associated excess cost of care. ${ }^{27}$

5) Vaccinations of Health Care Workers: Because of their frequent contact with patient or infective material from patients, many health-care workers (HCWs) (e.g., physicians, nurses, dental professionals, emergency medical personnel, laboratory technicians, medical and nursing students, hospital volunteers and administrative staff) are at risk of exposure to and possible spread of vaccinepreventable diseases. ${ }^{28}$ Conservation of immunity is, therefore, an essential part of prevention and infection control programs for HCWs. Optimum use of immunizing agents protects the health of 
workers and prevents patients from becoming infected through exposure to the infected worker. ${ }^{29}$ Consistent immunization programs could markedly reduce both the number of susceptible HCWs in hospitals and reduce the risks for transmission of vaccine-preventable diseases to other workers and patients. According to documented nosocomial transmission, HCWs are at a significant risk for acquiring or transmitting hepatitis B, influenza, measles, mumps, rubella and varicella. All of these diseases are vaccine-preventable. ${ }^{28} \mathrm{~A}$ study on effects of influenza vaccination of healthcare workers on mortality of elderly people in long-term care was associated with a substantial decrease in mortality among patients. ${ }^{30}$ Vaccination of health-care workers has been suggested as an additional or alternative strategy to lower rates of nosocomial transmission to patients at high risk of complications. ${ }^{31}$

6) Surveillance and control activities: Nosocomial infections (NI) surveillance, along with appropriate infection control activities, was able to decrease NI rates significantly while hospitals that did not use surveillance observed an increase in NI rates. ${ }^{32}$ This formed the basis for the development of the National Nosocomial Infections Surveillance (NNIS) system in the USA and also stimulated the establishment of national NI surveillance systems in various European countries, including France, the UK, Germany and various other countries. Patients infected with antimicrobial-resistant bacteria have significant morbidity and mortality and incur economic costs to healthcare facilities. ${ }^{33}$ Monitoring and reporting true nosocomial infections rates are important to control nosocomial infections. Specifically, an infection control physician, an infection control nurse for every 50 beds, organized surveillance and controlled activities and a system for reporting surgeon-specific infection rates were associated with a reduction in a hospital's infection rates by $32 \%{ }^{32}$ Results from in-hospital surveillance, estimate that almost $25 \%$ of all NIs are related to post-operative surgical site infections (SSI). Nonetheless, the magnitude of this problem is underrated, since $19 \%$ to $65 \%$ of SSIs are first diagnosed only after a patient's discharge from the hospital. ${ }^{34}$ Post-discharge nosocomial wound infections prolong morbidity and recovery time and may result in the patient's readmission to the hospital. ${ }^{34}$ So, it is necessary to perform some type of post-discharge surveillance to detect SSIs. Patients are more likely to suffer nosocomial infections if they are severely ill, if they are in the ICU or hospital for a prolonged time, have become colonized with resistant microorganisms, or have their host defenses breached by disease or instrumentation. Every day intensive care clinicians have to decide how best to integrate clinical and microbial information from these patients. $^{32}$

7) Antibiotic stewardship Healthcare-associated infections (HAIs) and anti-microbial resistance are well known major public health threats. Antibiotics have been used as an infection control measure for many years. With the widespread emergence of nosocomial antibiotic-resistant infections over the last decade, control of antibiotic use have become a priority for infection control. Therefore, monitoring antimicrobial consumption in hospitals is important in order to correlate a relationship with the development of resistance. ${ }^{35}$ Reason for increase in antibiotic resistance is the widespread use of antibacterial drugs. More than half of the patients in the acute care hospital receive antibiotics as treatment or prophylaxis. Hospital doctors often prescribe antibiotics excessively and inappropriately as shown in many studies. ${ }^{35}$ Broad-spectrum and the newer antibiotics should be reserved for specific patients and indications. ${ }^{36}$ Inadequate training in infectious diseases and antibiotic treatment, the difficulty of selecting the appropriate anti-infective drugs, insufficient use of microbiological information are pushing the use of broad-spectrum drugs. ${ }^{35}$ Despite national and local guidelines for antimicrobial therapy hospital doctors might be more prone to prescribe broad-spectrum antimicrobials for empirical treatment of bacterial infections. Additionally, they might be under pressure to produce speedy recoveries and thus less likely to step down therapy to narrow-spectrum antimicrobials. ${ }^{17,18}$ Use of monotherapy rather than combination therapy may favor the development of resistance in certain infections as will inadequately high drug doses or an improper route of administration, which could fail to achieve antibacterial drug levels at the site of infection. In order to limit the use of antibiotics, all requests for an antibiotic should be checked with an infectious diseases physician who decides whether use is appropriate, ${ }^{37}$ pharmacists should supervise the use of certain antibiotics and make recommendations for changes to the prescriber. Guidelines describing appropriate and inappropriate use of antimicrobials should be disseminated to the physicians and use of one agent should be replaced by another agent with similar spectrum of activity. ${ }^{38}$

8) Working environment and care coordination: Adverse psychosocial work conditions in the work stress models of job-demand-controlsupport, effort-reward imbalance and organizational justice have been shown to affect well-being and mental health of workers. This relationship is also true in case of healthcare professionals. Working conditions influence patient outcomes and healthcare quality. Reduced workload and shorter work hours, frequent provider training and computerized systems result in higher quality of patient care. Staffing is a key factor of healthcare-associated infection in critically ill patients. A substantial proportion of all infections could be avoided if nurse staffing were to be maintained at a higher level. ${ }^{39}$ Long work hours, high work stress and poor collaboration among the ward staff are associated with hospitalassociated infection among patients. Healthcare-associated infections are less likely in favorable critical care work environments. Focus on the quality of the work environment is a way to minimize the frequency of healthcare-associated infections. ${ }^{39}$

9) Communication, training and feedback

Keeping up-to-date with the latest findings regarding the spread of infections and strategies for prevention is crucial for a successful infection prevention program. "Infection preventionists must continually monitor the professional literature and attend educational conferences for the latest information with preventing infections. An intervention focused on the education of healthcare providers on the prevention of catheter-associated bloodstream infections decreased the incidence of primary bloodstream infections. Education programs lead to a considerable decrease in medical-care costs and patient morbidity caused due to central venous catheterization when implemented as part of mandatory training. Appreciating all the departments that support the infection prevention program and application of Comprehensive Unit-based Safety Programs intervention have markedly decreased SSI in a high-risk population. ${ }^{40}$

\section{CONCLUSION}

Hospital-acquired infections are by far the most common complication affecting hospitalized patients which has significant clinical and health economic burden on the healthcare system. The incidences of hospitalacquired infections caused by antibiotic-resistant pathogens have led to increases in morbidity and mortality. Development of resistance occurs from the interaction of microorganism, patients, hospital environment, including antibiotic use and infection control practices. An important 
cause of development of antibiotic resistance is due to the haphazard use of antibiotics. Further, transmission of resistant bacteria within hospitals by cross-colonization of patients via the hands of healthcare workers leads to the increase of hospital acquired infections. Strategies to control hospital acquired infections involves coordination among hospital administrator, clinicians, infectious disease specialist, microbiologist, hospital pharmacist, nurses; Implementing policies on use of antibiotics and infection control measures and being compliant towards it; Timely detection and reporting of antibiotic-resistant strains and improved surveillance.

\section{CONFLICT OF INTEREST}

The authors declare no conflict of interest.

\section{REFERENCES}

1. Reghu R, Vijayan M, Krishnan S. Nosocomial infections-An Overview. Int J Pharm Chem Biol Sci. 2014;4(2):291-7.

2. Brusaferro $S$, Arnoldo L, Cattani G, et al. Harmonizing and supporting infection control training in Europe. J Hosp Infect. 2015;89(4):351-6.

3. Allegranzi B, Nejad SB, Combescure C, et al. Burden of endemic health-careassociated infection in developing countries: Systematic review and metaanalysis. The Lancet. 2011;377(9761):228-41.

4. Shah S, Singhal T. Hand hygiene and health care associated infections: What, why and how. J Adv Nurs. 2013;5(3):130-4

5. Haley RW, Culver DH, White JW, et al. The efficacy of infection surveillance and control programs in preventing nosocomial infections in US hospitals. Am J Epidemiol. 1985;121(2):182-205.

6. Cruickshank R, Duguid JP, Marmion BP, Swain RHA. Medical microbiology a guide to the laboratory diagnosis and control of infection-v. 1: Microbial infections.-v. 2: The practice of medical microbiology-12. FAO. 1973;5-80

7. Festary A, Kouri V, Correa CB, Verdasquera D, Roig T, Couret MP. Cytomegalovirus and herpes simplex infections in mothers and newborns in a Havana maternity hospital. MEDICC Review. 2015;17(1):29-34.

8. Taylor K. Improving patient care by reducing the risk of hospital acquired infection: A progress report by the National Audit Office: Sage Publications Sage UK: London, England. 2004; 5-60.

9. Allegranzi B, Gayet-Ageron A, Damani N, et al. Global implementation of WHO's multimodal strategy for improvement of hand hygiene: A quasi-experimental study. Lancet Infect Dis. 2013;13(10):843-51.

10. Shah S, Singhal T. Hand hygiene and health care associated infections: What why and how. Pediatr Infect Dis. 2013;5(3):130-4.

11. Sharma R, Sharma M, Koushal V. P134: Hand washing: A critical measure in prevention and infection control. Antimicrob Resist Infect Control. 2013; 2(S1):P134.

12. Ampofo B. Healthcare workers' hand hygiene and infection control compliance: Should patients and relatives be involved?. J Infect Prev. 2013;14(6):214-8.

13. Sax H, Allegranzi B, Uckay I, Larson E, Boyce J, Pittet D. My five moments for hand hygiene": A user-centred design approach to understand, train, monitor and report hand hygiene. J Hosp Infect. 2007;67(1):9-21.

14. Jane WD. Hand adornment and infection control. Br J Nurs. 2007;16(11):654-6.

15. World Health O. WHO guidelines on hand hygiene in health care. First Global Patient Safety Challenge Clean Care is Safer Care. 2009. Retrieved from whqlibdoc. who. int/publications/2009/9789241597906_eng. pdf. 2009.

16. Mayfield JL, LeetT, Miller J, Mundy LM. Environmental control to reduce transmission of Clostridium difficile. Clin Infect Dis. 2000;31(4):995-1000.

17. Murray BE. Vancomycin-resistant enterococcal infections. N Engl J Med. 2000;342(10):710-21.

18. Johnson S, Gerding DN. Clostridium difficile-associated diarrhea. Clin Infect Dis. 1998;26:1027-34.

19. Maki DG, Alvarado CJ, Hassemer CA, Zilz MA. Relation of the inanimate hospital environment to endemic nosocomial infection. N Engl J Med. 1982;307(25):1562-6.
20. Brady MT, Evans J, Cuartas J. Survival and disinfection of parainfluenza viruses on environmental surfaces. Am J Infect Control. 1990;18(1):18-23.

21. Caul EO. Small round structured viruses: Airborne transmission and hospital control. The Lancet. 1994;343(8908):1240-2.

22. Stetson MG, Vautour RJ. Cost Effectiveness of Screening for MethicillinResistant Staphylococcus aureus (MRSA) and Vancomycin-Resistant Enterococcus (VRE) in an Intensive Care Unit at a 200-Bed Community Hospital with Low Prevalence of These Antibiotic-Resistant Organisms. Am J Infect Control. 2006;34(5):E40-1.

23. Harbarth S. Control of endemic methicillin-resistant Staphylococcus aureusrecent advances and future challenges. Clin Microbiol Infect. 2006;12(12):1154-62.

24. Nyman JA, Lees $\mathrm{CH}$, Bockstedt LA, et al. Cost of screening intensive care unit patients for methicillin-resistant Staphylococcus aureus in hospitals. Am J Infect Control. 2011;39(1):27-34.

25. Pogorzelska M, Stone PW, Larson EL. Wide variation in adoption of screening and infection control interventions for multidrug-resistant organisms: A national study. Am J Infect Control. 2012;40(8):696-700.

26. Jones M, Nielson C, Gupta K, Khader K, Evans M. Collateral benefit of screening patients for methicillin-resistant Staphylococcus aureus at hospital admission: Isolation of patients with multidrug-resistant gram-negative bacteria. Am J Infect Control. 2015;43(1):31-4.

27. Antwerpen CV, Caffrey A, Clement E, Gagnon C, Keehfus C. Cost-Effectiveness Assessment of Potential Financial Savings from Selective Culture Screening as a Strategy To Prevent Nosocomial Transmission of Methicillin-Resistant Staphylococcus aureus (MRSA) in 25 Upstate New York Hospitals. Am J Infect Control. 2006;34(5):E41.

28. Advisory Committee on Immunization P. Immunization of health-care personnel: Recommendations of the Advisory Committee on Immunization Practices (ACIP). MMWR. Recommendations and reports: Morbidity and mortality weekly report. Recommendations and Reports. 2011;60(RR-7):1.

29. Elder AG, O'Donnell B, McCruden EAB, Symington IS, Carman WF. Incidence and recall of influenza in a cohort of Glasgow healthcare workers during the 1993â€" 4 epidemic: Results of serum testing and questionnaire. BMJ. 1996:313(7067):1241-2.

30. Carman WF, Elder AG, Wallace LA, et al. Effects of influenza vaccination of health-care workers on mortality of elderly people in long-term care: A randomised controlled trial. The Lancet. 2000;355(9198):93-7.

31. Elder AG, O'Donnell B, McCruden EAB, Symington IS, Carman WF Incidence and recall of influenza in a cohort of Glasgow healthcare workers during the 1993-4 epidemic: Results of serum testing and questionnaire. BMJ. 1996;313(7067):1241-2

32. Haley RW, Culver DH, White JW, et al. The efficacy of infection surveillance and control programs in preventing nosocomial infections in us hospitals. Am J Epidemiol. 1985;121(2):182-205.

33. Jarvis WR. Selected aspects of the socioeconomic impact of nosocomial infections: Morbidity, mortality, cost and prevention. Infect Control Hosp Epidemiol. 1996;17(8):552-7.

34. HoltzTH, Wenzel RP. Post discharge surveillance for nosocomial wound infection: A brief review and commentary. Am J Infect Control. 1992;20(4):206-13.

35. Gould IM, Hampson J, Taylor EW, Wood MJ. Hospital antibiotic control measures in the UK. J Antimicrob Chemother. 1994;34(1):21-42.

36. Cars O, Molstad S, Melander A. Variation in antibiotic use in the European Union. The Lancet. 2001;357(9271):1851-3.

37. Climo MW, Israel DS, Wong ES, Williams D, Coudron P, Markowitz SM. Hospitalwide restriction of clindamycin: Effect on the incidence of Clostridium difficileassociated diarrhea and cost. Ann Intern Med. 1998;128(12_Part_1):989-95.

38. Bradley SJ, Wilson ALT, Allen MC, Sher HA, Goldstone AH, Scott GM. The control of hyperendemic glycopeptide-resistant Enterococcus spp. on a haematology unit by changing antibiotic usage. J Antimicrob Chemother. 1999;43(2):261-6.

39. Kelly D, Kutney-Lee A, Lake ET, Aiken LH. The critical care work environment and nurse-reported health care-associated infections. Am J Crit Care. 2013;22(6):482-8

40. Wick EC, Hobson DB, Bennett JL, et al. Implementation of a surgical comprehensive unit-based safety program to reduce surgical site infections. J Am Coll Surg. 2012;215(2):193-200.

Article History: Submission Date : 06-02-2020 ;Revised Date : 24-02-2020; Acceptance Date : 29-02-2020

Cite this article: Arpan S, Kalyan R, Mainak C, Karunakaran G. Correlation between Antimicrobial Resistance and Nosocomial Infections, their Prevention and Control: Review. J Young Pharm. 2020;12(1):25-8 